\title{
Archéopages
}

Archéopages

Archéologie et société

$44 \mid 2017$

Terrains vagues

\section{Le Marais-Vert à Strasbourg.}

Un espace intra-muros peu urbanisé, XIII ${ }^{\mathrm{e}}$-XIX ${ }^{\mathrm{e}}$ siècle

Le Marais-Vert in Strasbourg.A not very urbanized intra-urban space, $13^{\text {th }}-19^{\text {th }}$ century

El Marais-Vert de Estrasburgo. Un espacio intramuros poco urbanizado, siglos XIII a XIX

\section{Catherine Xandry}

\section{(2) OpenEdition}

Journals

Édition électronique

URL : https://journals.openedition.org/archeopages/1510

DOI : $10.4000 /$ archeopages. 1510

ISSN : 2269-9872

Éditeur

INRAP - Institut national de recherches archéologiques préventives

Édition imprimée

Date de publication : 1 juin 2017

Pagination : 40-47

ISSN : 1622-8545

Référence électronique

Catherine Xandry, "Le Marais-Vert à Strasbourg. », Archéopages [En ligne], 44 | 2017, mis en ligne le 01 juillet 2019, consulté le 03 juin 2021. URL : http://journals.openedition.org/archeopages/1510 ; DOI : https://doi.org/10.4000/archeopages.1510 


\section{Le Marais-Vert à Strasbourg Un espace intra-muros peu urbanisé, $\mathrm{XIII}$-XIX ${ }^{\mathrm{e}}$ siècle

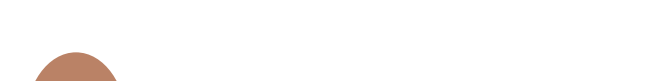

Catherine Xandry Universitede de Picarrie, EA 4284, «TRAME»

40

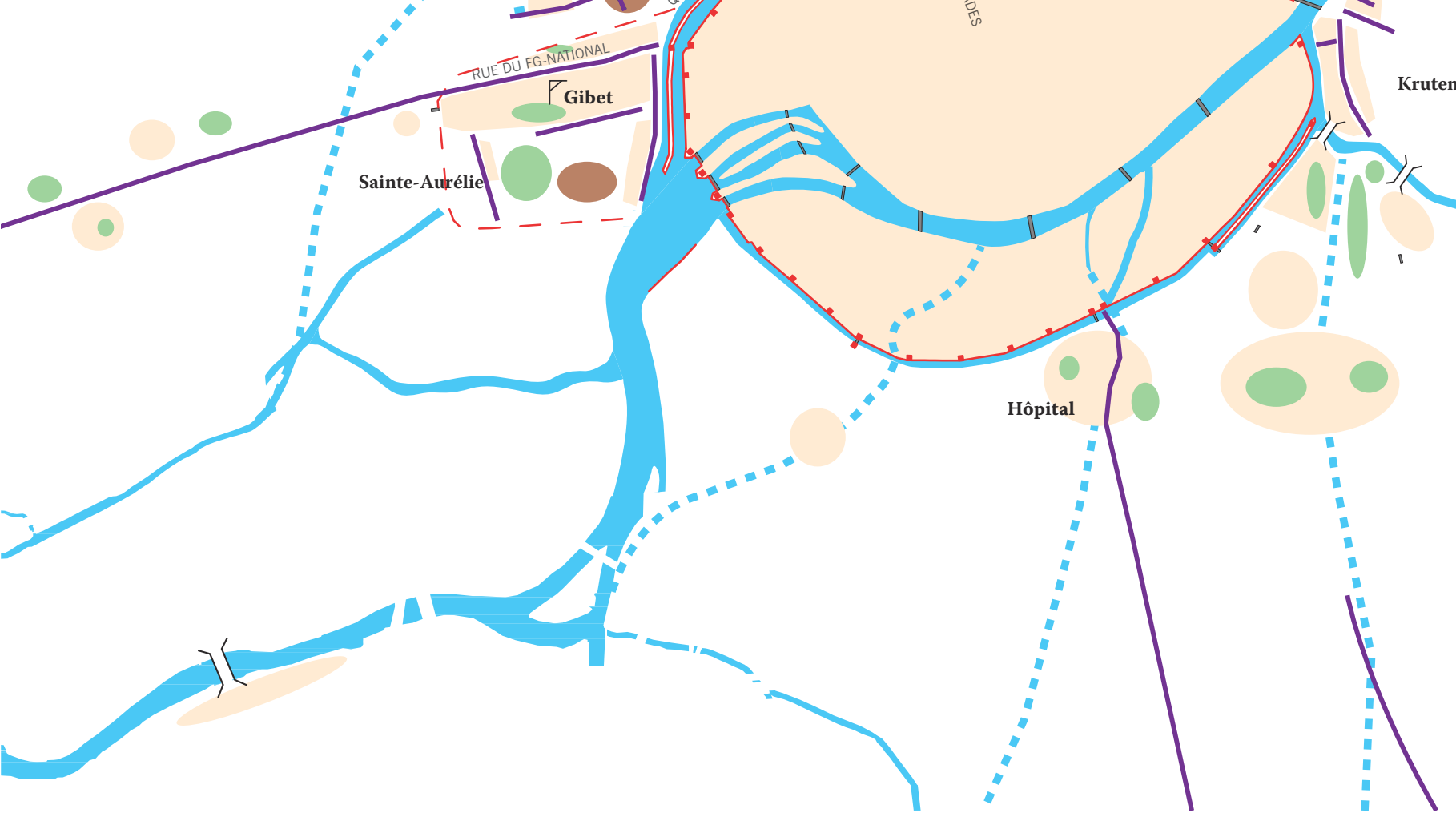




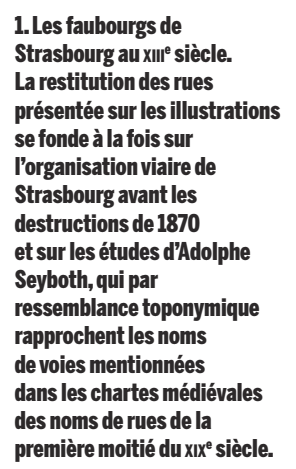

La ville de Strasbourg, dans son évolution depuis l'Antiquité jusqu'au $\mathrm{XIX}^{\mathrm{e}}$ siècle, a vu un espace particulier être intégré à son enceinte du $\mathrm{XIV}^{\mathrm{e}}$ siècle. En effet, le quartier dit du Marais-Vert (Grüne Bruch), toponyme en relation avec le caractère marécageux du lieu depuis l'Antiquité, est inclus dans l'enceinte alors qu'il semble rester totalement vide. Quelle est alors sa fonction dans l'organisation de la ville ? Cet article, issu d'une thèse (Xandry, 2013), va essayer de répondre à cette question en s'attachant à décrire l'évolution de cet espace du XIII ${ }^{\mathrm{e}}$ au XIX ${ }^{\mathrm{e}}$ siècle, en distinguant les espaces ouverts des espaces bâtis et, au sein de cette répartition, les occupations pérennes des usages temporaires.

Précisément, le lieu-dit du Marais-Vert correspond plus ou moins à l'espace compris entre les actuels fossé du Faux-Rempart, rue du Faubourg-de-Saverne, rue de Sébastopol et boulevard du Président-Wilson. Cependant, l'espace étudié ici sera un peu plus vaste. En effet, bien que ce toponyme ait servi de principal repère topographique pour ce quartier dans les chartes médiévales, avec le Marais-Kageneck (Kageneckerbrüch) qui le jouxte, il s'intègre en fait dans un espace « vide » qui s'insère entre les deux voies d'accès principales à l'ouest de la ville : les actuelles rues du Faubourg-National et du Faubourg-de-Pierre, anciennes voies romaines le long desquelles se sont développées des nécropoles antiques, puis des faubourgs. Ces deux faubourgs

1. Prétendument érigée sur la tombe de saint Arbogast. 2. D'après les

Urkundenbücher. 3. Littéralement : livres/recueils d'actes, de chartes ; édition des chartes médiévales de Strasbourg jusqu'en 1400 : Fritz, 1899 ;

Schulte, 1884 ;

Schulte, Wolfram, 1888 ;

Wiegand, 1879 ; Wiegand,

1886; Wiegand, 1898 ;

Witte, 1900 ;

Witte, Wolfram, 1896.

4. Interprétation

renforcée par la

découverte, au 43 rue

Kageneck, de bâtiments

agricoles pouvant

appartenir à un corps de

ferme (XIV $-X^{\mathrm{e}}$ siècles)

(Keller, 1996, p. 39).

5. Peut-être vers l'actuel

square des Halles d'après

le plan Merian de

Strasbourg de 1663

(Die Statt Strassburg wie

sie jetziger Zeit im wesen

steht A.o 1643),

Bibliothèque nationale

et universitaire

de Strasbourg (BNUS),

M.CARTE.100.603.

6. D'après l'inscription

« Diss Werk Word

gemacht und vollbracht

uf den nächsten Freytag

von mitfasten des Jahres

als man zalt nach Gottes Geburth MCCCCXXII ». étuves, boulangerie, hospice, chapelle et auberges. Entre ces deux faubourgs, ces deux voies d'accès à la ville, s'étend un espace à première vue vide [ill. 2].

\section{Un espace non bâti mais occupé...}

À partir du XIII ${ }^{\mathrm{e}}$ siècle, le Marais-Vert est délimité, à l'ouest, par l'extension de la ville (entre 1200 et 1228), à l'est, par la porte du faubourg de Pierre, et au nord, par l'installation d'une tour de guet vers le milieu du XIv ${ }^{\mathrm{e}}$ siècle, au milieu de la ligne joignant la porte des deux faubourgs.

De prime abord, il se présente comme un espace rural : les Urkundenbücher ${ }^{3}$ mentionnent en effet plusieurs champs ( 20 avant 1374 et 3 après l'extension de l'enceinte), une cour agricole (le Kageneck $)^{4}$, quelques maisons individuelles et quelques jardins. On distingue un petit hameau vers la rue Kageneck, comprenant de nombreux espaces ouverts (champs et jardins) [ill. 3]. On repère également des éléments structurant l'espace. Il s'agit tout d'abord de la chapelle de la Croix-des-Miséreux (Ellenden Crutze), érigée au XIII ${ }^{\mathrm{e}}$ siècle ${ }^{5}$, dernière étape des condamnés avant le gibet. La localisation de ce dernier est compliquée. Néanmoins, on peut émettre l'hypothèse qu'au XIII ${ }^{e}$-XIV ${ }^{\mathrm{e}}$ siècle, il se situait dans les environs du Marais-Vert. Son histoire est difficile à percevoir. Si les cartes de l'époque moderne indiquent le lieu-dit justice extra-muros, à la hauteur de Cronenbourg, le gibet érigé là ne daterait que de $1422^{6}$. Par ailleurs, on sait que l'évêque saint Arbogast (mort vers 675) aurait demandé à se faire enterrer près du gibet. Si on accepte le fait que celui-ci fut enterré là où sera érigée la chapelle Saint-Michel, cela voudrait dire que le gibet se trouvait alors à proximité de l'actuelle église Sainte-Aurélie. En revanche, un certain nombre d'éléments permettent d'émettre l'hypothèse de la localisation du gibet au MaraisVert au XIII ${ }^{\mathrm{e}}$-XIV ${ }^{\mathrm{e}}$ siècle. Il y a tout d'abord la présence de la chapelle de la Croix-des-Miséreux ; puis l'existence d'une rue du Gibet (Galgenstrasse), mentionnée en 1251 et identifiée par Adolphe Seyboth comme étant l'actuelle rue des Païens. Enfin, au XVI ${ }^{\mathrm{e}}$ siècle, le bourreau habite au MaraisVert (Seyboth, 1890, p. 268). On aurait donc à cette période un gibet qui se serait décalé vers l'est depuis le haut Moyen Âge, sans s'être encore fixé à son emplacement de l'époque moderne et qui ne semble pas alors être sur un axe important de circulation. Autour du lieu choisi pour la place du gibet, occupé de façon épisodique, se trouvent des espaces de cultures entretenus sur la durée.

\section{... Et qui fait partie de la ville}

Les menaces que subit Strasbourg à la fin du $\mathrm{XIV}^{\mathrm{e}}$ et dans le courant du $\mathrm{XV}^{\mathrm{e}}$ siècle l'encouragent à agrandir son enceinte et à protéger ses faubourgs les plus importants. La première étape de cette extension est la construction de l'enceinte ouest entre 1374 et 1390. Elle réunit en une seule entité spatiale les zones de Sainte-Aurélie et du faubourg 

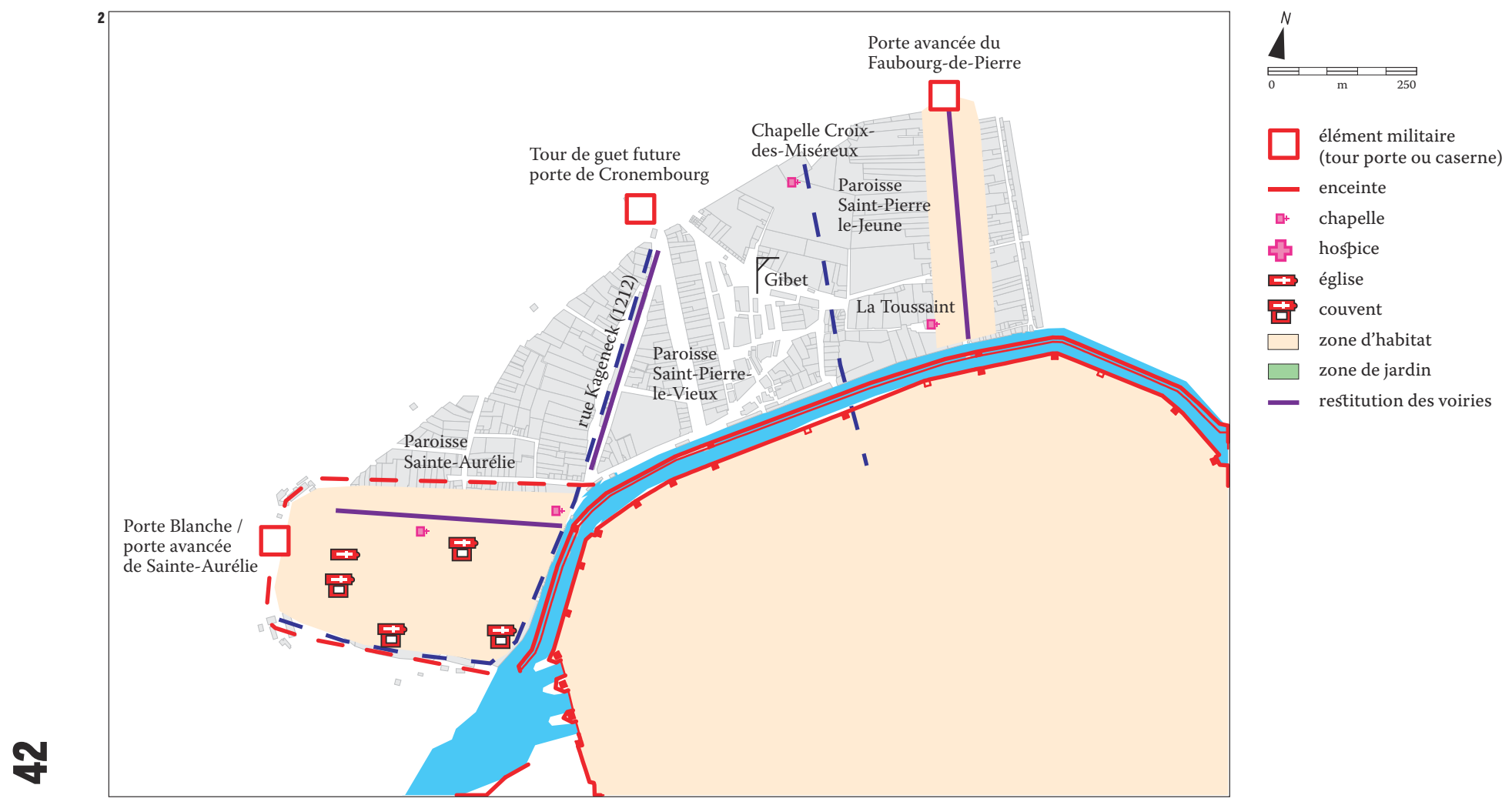

- enceinte

ㅁ. chapelle

hospice

드 église

표 couvent

$\square$ zone d'habitat

$\square$ zone de jardin

restitution des voiries

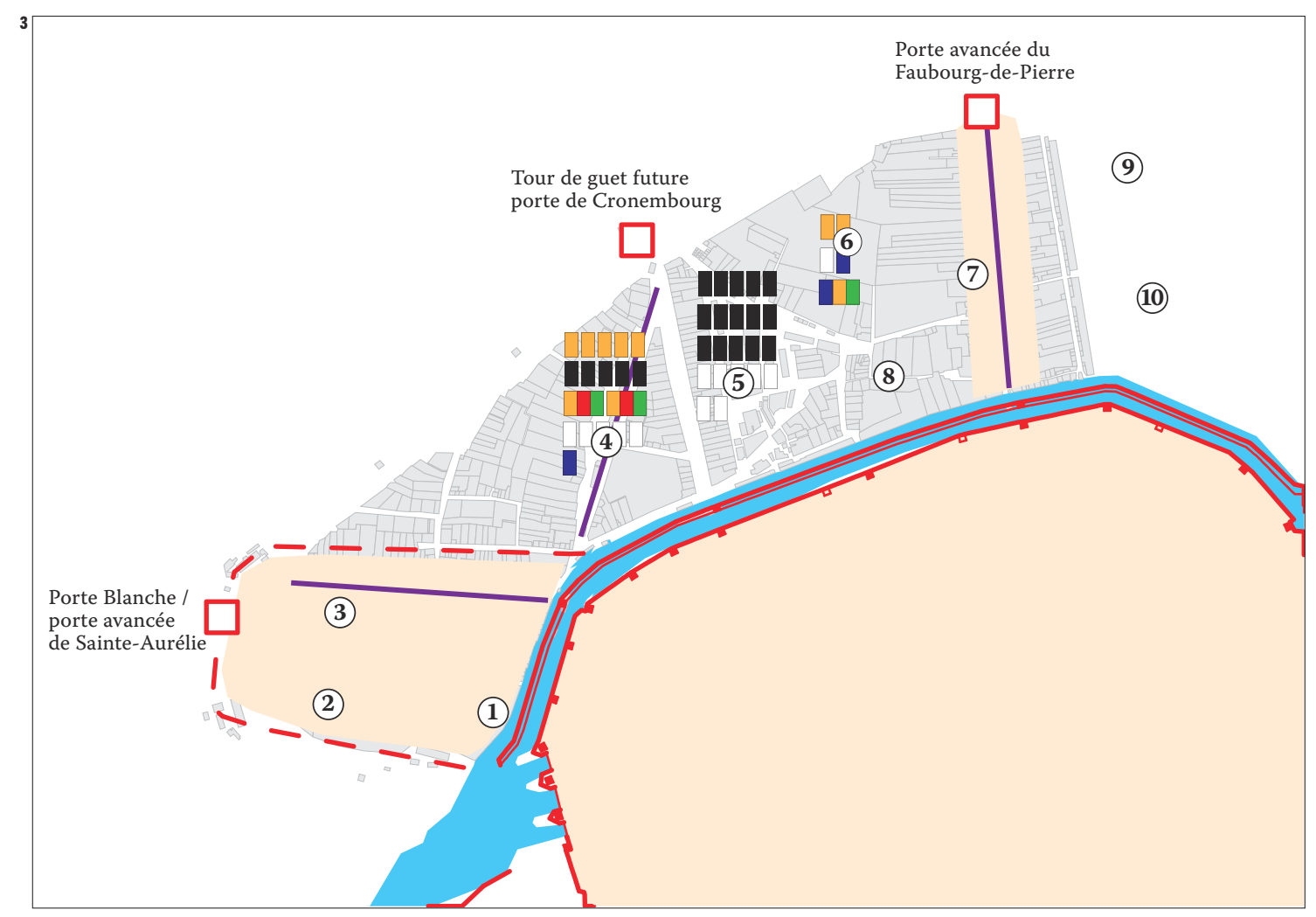
1. Grüneworth
2. Teutsch Au
6. Wüste Bruch
3. Sainte-Aurélie
7. Stein Strasse
4. Kagenecker Bruch
8. Aller Heiligen Bruch
9. Rosengarten
5. Grüne Bruch
10. Im Rausch

cour

jardin

$\square$ description du bien inconnue 

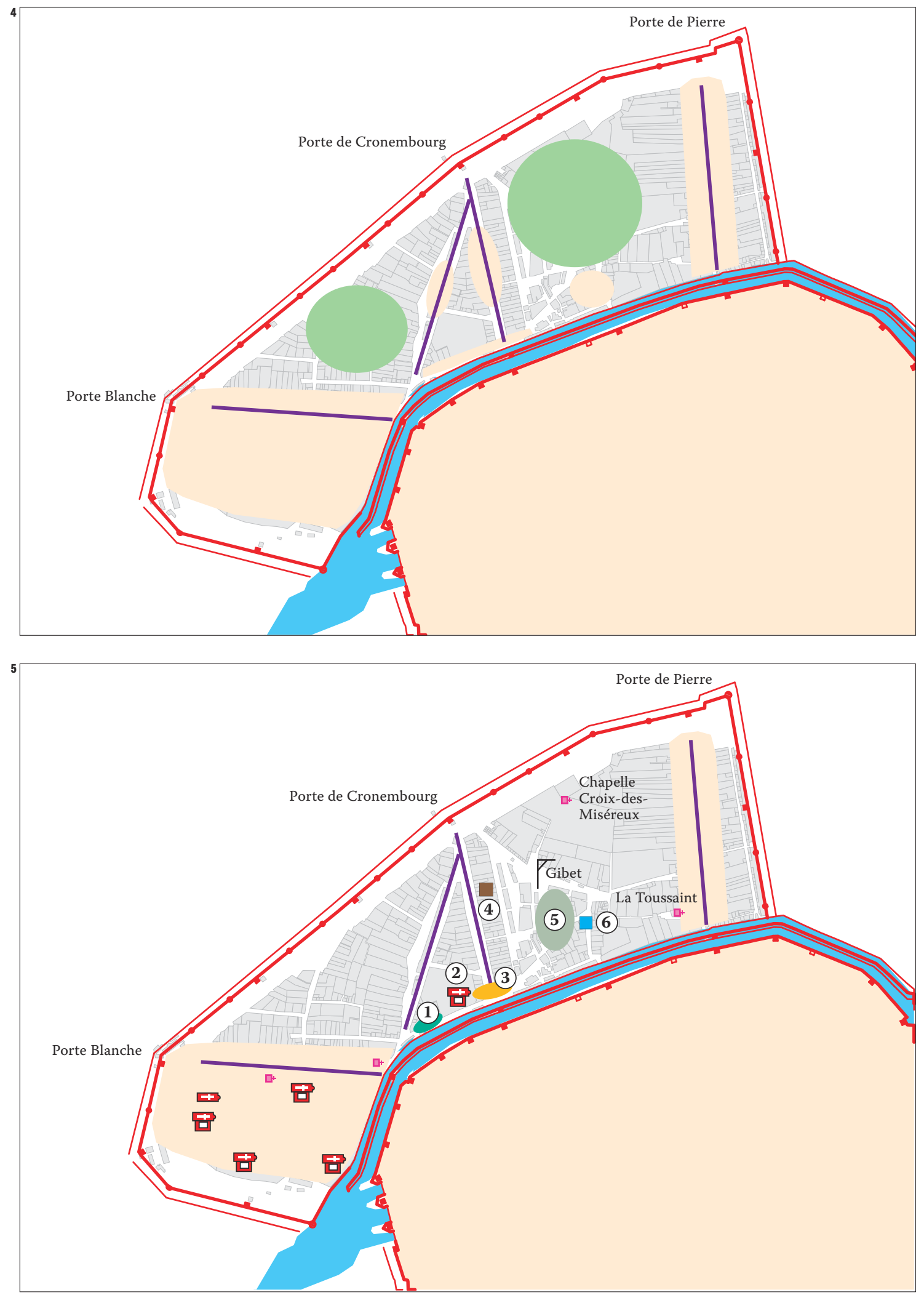

1. marché au bois

2. Saint-Marc et Saint-Jean

4. arsenal

3. dépotoir

5. champ de tir

6. maison du bourreau
2. Organisation des faubourgs ouest de Strasbourg au

XIII'-XIVe siècle, avant la

construction de l'enceinte

entre 1374 et 1390 . Le fond

de plan utilisé principalement

pour les illustrations de cet

article a été redessiné sur

le plan de Strasbourg de

Blondel, réalisé vers 1765

(Archives départementales du

Bas-Rhin,1L/PLAN5/1 à 1L/

PLAN5/10). Ceci permet de

travailler sur un état des rues

antérieur aux grandes

destructions de 1870 qui ont

remodelé profondément

l'organisation des quartiers

étudiés ici (disparition

de certaines rues et création

de nouvelles).

3. Biens mentionnés au

Marais-Vert dans les

Urkundenbïcher entre 1200

et 1374. Le plan se fonde sur

les chartes (entre 1200 et

1374) d'achat ou de location

de biens (champs, cours,

maisons... ou association

de plusieurs éléments) situés

ou associés à un toponyme.

Ne pouvant associer

précisément un bien à une

parcelle déterminée, le plan

se contente d'indiquer les

types de biens, ainsi que leur

fréquence dans les sources.

Cela permet de restituer

une concentration respective

plus importante de champ

au Marais-Vert et un hameau

au Marais Kageneck.

4. Habitats et espaces

ouverts au Marais-Vert

au $x v^{e}$ et xvie siècle. L'extension

de l'enceinte, à l'ouest de la

ville, entre 1374 et 1390 ,

s'appuie sur des éléments

préexistants : les portes

avancées de Sainte-Aurélie

et du faubourg de Pierre,

ainsi que sur la tour de guet

entre les deux, laquelle

devient alors porte de

Cronembourg.

5. Structures présentes

au Marais-Vert au xve

et xvI ${ }^{e}$ siècle. 


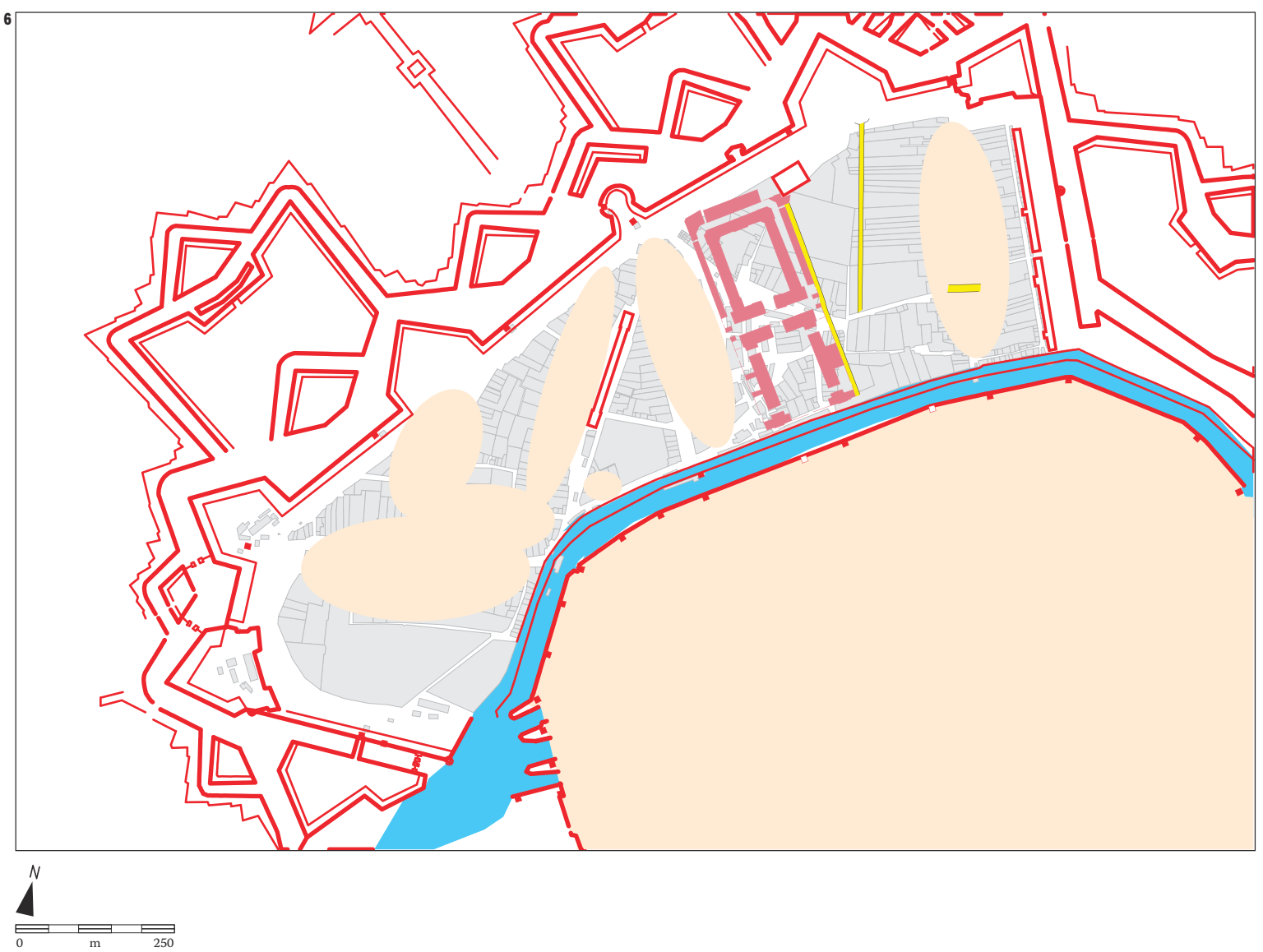

de Pierre, ajoutant 76 hectares à la superficie initiale de l'enceinte. Il s'agit là de fortifier le côté de la ville le plus vulnérable aux attaques et surtout deux des principales voies d'accès à la ville, ainsi que des quartiers artisanaux possédant église et couvents. Elle englobe également tout l'espace cultivé entre ces deux faubourgs, en s'appuyant sur les portes avancées de Sainte-Aurélie et du faubourg de Pierre, ainsi que sur la tour de guet entre les deux, laquelle devient alors porte de Cronembourg. Bien que largement constitués d'espaces ouverts, le Marais-Vert et le Kageneck font alors partie intégrante de la ville intra-muros et deviennent, grâce à la porte de Cronembourg et à la rue de Saverne qui la relie à la cité, le troisième axe d'accès ouest à la ville. Petit à petit, les champs vont laisser la place aux jardins maraîchers. L'habitat, bien qu'il soit difficile d'en juger au travers des sources textuelles, reste peu dense, dispersé et a plutôt tendance à s'installer le long des quais du fossé du Faux-Rempart [ill. 4].

$\mathrm{Si}$ le gibet reste très certainement situé dans cet espace au moins jusqu'en 1422, et que la chapelle de la Croix-des-Miséreux est toujours attestée, d'autres éléments apparaissent au MaraisVert au $\mathrm{XV}^{\mathrm{e}}$ siècle, comme le champ d'exercice des arbalétriers, ainsi qu'un arsenal. Se pose alors la question de la façon dont les terrains nécessaires à la mise en place de ces structures sont acquis. En effet, si la construction de l'arsenal ne nécessite certainement qu'une surface restreinte, facilement achetable par la ville, la mise en place d'un champ de tir nécessite une surface relativement importante et donc, peut-être, l'acquisition de plusieurs parcelles juxtaposées. Cependant, si l'on observe l'état du parcellaire en 1765 sur le plan Blondel, on constate l'existence, encore au $\mathrm{XVIII}^{\mathrm{e}}$ siècle, de parcelles de très grandes dimensions. Le champ de tir s'est donc peut-être installé sur une seule parcelle. Dans tous les cas, il a une durée de vie relativement limitée puisqu'il est déplacé dès 1480 et à plusieurs reprises par la suite (sans que l'on sache pourquoi) avant d'être définitivement fixé, en 1540, au Contades (lieu-dit Waseneck). L'arsenal disparaît en 1545.

Sur ces vastes parcelles périphériques se développent deux activités pérennes sur plusieurs générations. Un marché au bois apparaît au lieu-dit « Marais-Vert » à partir de 1466 ; le bois d'œuvre était amené par flottage par le fossé extérieur de l'enceinte du XIII ${ }^{\mathrm{e}}$ siècle (Schwien, 1992, p. 148) [ill. 5]. Un dépotoir, repéré lors de fouilles ${ }^{7}$, reçoit entre le $\mathrm{XIV}^{\mathrm{e}}$ et le $\mathrm{XVI}^{\mathrm{e}}$ siècle (principalement entre 1350 et 1500), près de 1 à 1,50 m de déchets sur la berge du Faux-Rempart de part et d'autre de la rue du Faubourg-de-Saverne (Baudoux et al., 1994, p. $160-161)^{8}$; c'est-à-dire à proximité immédiate des zones d'habitat les plus denses de cet espace, au sortir de l'ancienne ville intra-muros et le long d'un axe important de circulation. Si aucune limite n'a été relevée lors de la fouille, il pourrait s'étendre sur un quadrilatère de $100 \mathrm{x} 300 \mathrm{~m}$, soit près de
7. Fouilles des Halles, quai Saint-Jean, quai Kléber, 1991-1992, sous la direction de JeanJacques Schwien, Afan. 8. La transformation de ce site en dépotoir est peut-être liée à la mise en place de l'enceinte, peut-être en raison du manque de place dans le noyau urbain. Deux dépotoirs différents y ont été observés : sur le quai Saint-Jean, un amoncellement d'ossements d'animaux ; sur le quai Kléber, beaucoup d'os, mais aussi des lentilles très organiques et de la céramique. 
3 ha. Le dépotoir est abandonné au milieu du $\mathrm{XVI}^{\mathrm{e}}$ siècle pour faire place à des habitations. On voit donc comment au « Marais-vert » l'espace est occupé par des activités continues ou périodiques, de type péri-urbain, en des lieux précis ou changeants.

Enfin, le paysage religieux subit plusieurs transformations. Tout d'abord, la destruction des couvents extra-muros de Saint-Jean-aux-Ondes et de Saint-Marc au moment de la mise en place du glacis (1475) entraîne la construction du nouveau couvent de dominicaines qui unit les deux anciennes communautés Saint-Marc et Saint-Jean. Ce dernier s'installe alors au MaraisVert, en bordure du fossé du Faux-Rempart. Le choix de cet emplacement est sans doute lié au fait que le couvent Saint-Marc y possédait déjà plusieurs parcelles ainsi qu'au faubourg de Pierre (12 d'après les Urkundenbücher et Seyboth, 1890 dont sept près de l'emplacement futur du couvent avant sa construction) ${ }^{9}$. Le Marais-Vert sert alors de zone de repli. Le couvent sera fermé à la suite de la Réforme en 1529, tout comme les chapelles de la Croix-des-Miséreux (1543) et de La Toussaint (1529).

\section{Un espace de potentialités \\ dans la ville $\left(\mathrm{XVII}^{\mathrm{e}}\right.$-XIX ${ }^{\mathrm{e}}$ siècle $)$}

Le passage de la ville à la France, en 1680, entraîne des transformations qui se répercutent au niveau du Marais-Vert. Devenue ville forte de frontière, devant de nouveau accepter le catholicisme dans ses murs, de nouvelles fonctions apparaissent.

Le couvent Saint-Marc et Saint-Jean sert à l'Assistance Publique jusqu'en 1687 avant que la messe n'y soit rétablie et que son église accueille le culte catholique de la paroisse Sainte-Aurélie (l'église Sainte-Aurélie demeurant un temple protestant). Le couvent en lui-même sert dorénavant aux johannites dont les bâtiments ont été détruits en 1633. La maison du bourreau est toujours située dans ce quartier, ainsi que plusieurs auberges, une boulangerie, un marché aux bois et des péages. Enfin, d'après le plan de 1744 (Bibliothèque nationale et universitaire de Strasbourg, M.CARTE.1.224), la transformation de la ville en « ville militaire » entraîne la création dans ce quartier de trois casernes, dont une caserne d'infanterie et une autre comportant également des écuries pour la cavalerie, et d'un magasin à poudre.

Le quartier reste cependant une zone maraîchère. Des dynasties de jardiniers y cultivent différentes plantes, dont le tabac à partir du XVII ${ }^{\mathrm{e}}$ siècle. Mais les particuliers exploitent également cet espace pour d'autres intérêts. En effet, depuis le décret du 15 octobre 1810, relatif « aux manufactures et ateliers qui répandent une odeur insalubre ou incommode », l'installation en ville de différents types d'entreprises est soumise à l'approbation d'un conseil de salubrité.
Par exemple, les amidonniers, les abattoirs ou les fours à chaux doivent être strictement isolés de toute habitation. Dans cette optique, ce quartier est un lieu attractif, du fait de la faible densité du bâti tout en étant intra-muros.

Cependant, les particuliers ne sont pas les seuls à élaborer des projets. Les différentes institutions étatiques (Génie militaire, Ponts-et-Chaussées...) ou municipales (Service d'architecture...), qui apparaissent aux XVIII ${ }^{\mathrm{e}}$ et $\mathrm{XIX}^{\mathrm{e}}$ siècles et se partagent la gestion de l'espace de la ville, s'intéressent également à ce quartier et y superposent les projets. Ainsi, le plan Blondel, vers 1765 , présente le projet d'une grande caserne de cavalerie destinée à occuper l'espace du MaraisVert, et propose le percement de deux nouvelles rues desservant l'espace entre cette caserne et la rue du Faubourg-de-Pierre [ill. 6]. La municipalité a aussi des vues sur ce quartier. Au début du XIx ${ }^{e}$ siècle, il est question d'un remaniement urbanistique (un plan d'alignement), à l'occasion de nouvelles constructions : une caserne, une halle au blé et un port (Archives de la Ville et de l'Eurométropole de Strasbourg [AVES], $312 \mathrm{MW} 336,1811)$. De ce vaste projet, seule la halle au blé verra le jour (1825-1829). Mais la volonté édificatrice se poursuit : une usine à gaz en 18381839 puis une gare en cul-de-sac en 1854. En 1853, la municipalité projette le percement d'une rue en prolongement de la rue des Grandes Arcades, dans le quartier du Marais Vert, associée à la mise en place d'une cité ouvrière (AVES, $312 \mathrm{M} \mathrm{W}_{2}$ ) [ill. 7] ; un projet qui n'aboutira pas. On peut avancer deux hypothèses pour expliquer ce fait : soit la ville n'a pas réussi à acquérir les parcelles nécessaires à ses visées, soit le siège de 1870 a stoppé net toute réalisation [ill. 8].

En effet, le siège de 1870 et la destruction intégrale de tous les quartiers ouest de la ville (Faubourg-de-Pierre, Faubourg-National ou Sainte-Aurélie, Faubourg-de-Saverne...) ainsi que le changement d'autorité (et donc de vision urbanistique) chamboulent le développement du Marais-Vert. Les années suivantes voient une refonte totale de son organisation : agrandissement de l'enceinte, nouveau tracé de rues, nouvelle gare, remplacement des espaces ouverts par du bâti... Pour la première fois, un plan directeur général est imaginé.

Espace peu densément bâti jusqu’à la fin du XIX ${ }^{\mathrm{e}}$ siècle, mais inclus dans l'enceinte à partir du XIV siècle du fait de sa position entre deux faubourgs importants, le Marais-Vert reste principalement composé d'espaces de culture et de maraîchage. Il s'agit alors d'une particularité dans l'espace intra-muros de Strasbourg puisque le reste de la ville semble densément bâti ou, dans le cas de la Krutenau, présente un enchevêtrement bâti-espace ouvert plus complexe. Surtout dédié à des activités rurales, il voit se succéder des activités qui attendent de trouver un emplacement définitif
9. Saint-Marc n'était
d'ailleurs pas le seul établissement à posséder des biens dans ce quartier : on peut citer Saint-Pierre, La Toussaint... 

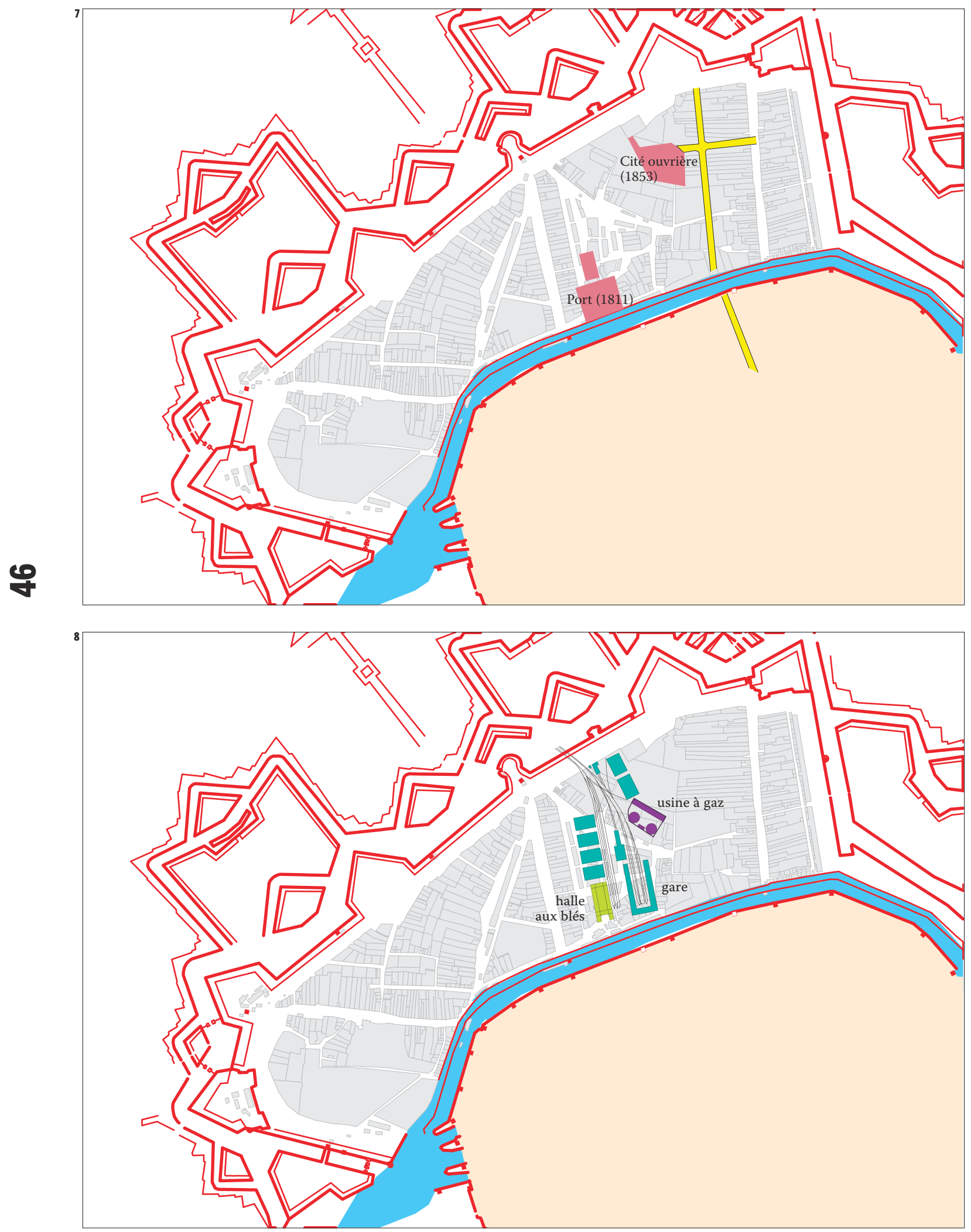

$\underline{1+}=$ 
dans la ville (arsenal, champ de tir, gibet, marché au bois). Finalement, que cela soit de façon consciente ou non, la ville (ou les autorités présentes en ville) semble l'utiliser comme un lieu de repli (comme pour Saint-Jean et SaintMarc en 1475), d'essai d'implantation de fonctions spécifiques (sans que celles-ci n'amènent au développement d'une zone d'habitat), de réserve ou de potentialités futures. Il s'agit là d'un espace qui est laissé « vierge » de réalisations urbanistiques de grande ampleur. Il rappelle en cela le concept de «fringe belt» développé par M. R. G. Conzen (Conzen, 1968). En effet, le Marais-Vert s'établit au départ comme une partie de la ceinture limitrophe de l'enceinte de la ville au XIII ${ }^{\mathrm{e}}$ siècle, avec une majorité de parcelles de grande taille. Est-ce dû au fait qu'il dépende d'une paroisse différente de celles des deux faubourgs qui l'entourent, à l'absence de couvents, de voies d'accès anciennes à la ville, à l'importance économique de ses jardins, au «poids » de la corporation des jardiniers, à une faible pression démographique qui n'a pas obligé la ville à construire plus d'habitat, même après la mise en place du glacis en 1475 qui stoppe l'extension extramuros, ou tout simplement à un désintérêt pour cet espace? Dans tous les cas, ce quartier semble correspondre à la manifestation physique d'un ralentissement de la croissance urbaine, avant un développement plus marqué au XVIII ${ }^{\mathrm{e}}$ puis $\mathrm{XIX}^{\mathrm{e}}$ siècle, ce qui correspond à la phase de fixation puis d'expansion de Conzen.
Même si l'évolution des archives au XVIII ${ }^{\mathrm{e}}$ et $\mathrm{XIX}^{\mathrm{e}}$ siècle permet de mieux percevoir les projets de la municipalité, rien n'empêche de penser que des réflexions similaires n'ont pas existé antérieurement. La grande taille des parcelles devait d'ailleurs sembler être un avantage économique pour ces projets, puisque ces derniers nécessitaient l'achat ou l'expropriation de moins de parcelles et surtout une négociation avec moins d'interlocuteurs. Cependant, tant qu'une étude précise, dans la longue durée, de la politique foncière des propriétaires des parcelles n'aura pas été faite, de même que l'analyse de la politique d'acquisition de parcelles par la ville pour ses projets, il sera difficile de répondre précisément à ces questions. Cette étape est d'autant plus nécessaire qu'elle permettra de comparer plus précisément le Marais-Vert à d'autres cas comme le Marais, en rive droite à Paris (Mirlou, Noizet, Robert, 2013), qui présente une utilisation comparable au Moyen Âge comme zone maraîchère mise notamment en place par le chapitre de Sainte-Opportune et par des particuliers, puis, par la suite, de zone de rejet de déchets. C'est alors la comparaison des stratégies foncières respectives des divers types d'acteurs (religieux, particuliers...) qui permettrait de mettre en lumière les spécificités de ce quartier strasbourgeois ou au contraire de valider son rattachement à un modèle.
Références bibliographiques

FRITZ J., 1899, Urkundenbuch des Stadt Strassburg. Sechster Band. Politische Urkunden von 1381 bis 14.oo, Strasbourg, Karl J. Trübner, 923 p.

SChulte A., 1884, Urkundenbuch des Stadt Strassburg. Dritter Band: Privatrechtliche Urkunden und Amtlisten von 1266 bis 1332, Strasbourg, Karl J. Trübner, $451 \mathrm{p}$.

SChulte A., Wolfram G., 1888, Urkundenbuch des Stadt Strassburg. Vierter Band zweite Hälfte. Stadtrechte und Aufzeichnungen über bischöflichStädtische und bischöfliche Ämter, Strasbourg, Karl J. Trübner, $309 \mathrm{p}$.

WIEG AND W., 1879, Urkundenbuch des Stadt Strassburg. Erster Band: Urkunden und Stadtrechte bis zum Jahr 1266, Strasbourg, Karl J. Trübner, 585 p.

Wieg AND W., 1886, Urkundenbuch des Stadt Strassburg. Zweiter Band: Politische Urkunden von 1266 bis 1332 Strasbourg, Karl J. Trübner, $482 \mathrm{p}$.

WIEG AND W., 1898, Urkundenbuch des Stadt Strassburg. Vierter Band erste Hälfte : Nachträge und Berichtigungen, Strasbourg, Karl J. Trübner, 360 p.

Wit te H., 190o, Urkundenbuch des Stadt Strassburg. Siebenter (Schluss-) Band. Privatrechtliche Urkunden und Rathslisten von 1332 bis 14.0o, Strasbourg, Karl J. Trübner, $1165 \mathrm{p}$.

Witte H., Wolfram G., 1896, Urkundenbuch des Stad Strassburg. Fünfter Band. Politische Urkunden von 1332 bis 1380, Strasbourg, Karl J. Trübner, 1128 p.
Baudoux J., et al., 1994, Strasbourg. 10 ans d'archéologie urbaine. De la caserne Barbade aux fouilles du Tramway. Catalogue de l'exposition présentée a Strasbourg du 15 octobre 1994 au 29 janvier 1995, Strasbourg, Musées de la ville de Strasbourg, 223 p.

Conzen M. R. G., 1968, « The Use of Town Plans in the Study of urban History », in Dyos H. J., The Study of Urban History (proceedings of an international round-table conference of the urban History Group in Leicester, 23-26 september 1966), Londres, Edward Arnold, p. 113-129.

Keller M., 1996, « Strasbourg, 43 rue Kageneck », Bilan scientifique régional Alsace, Service régional de l'Archéologie, p. 39.

Mirlou L., Noizet H., Robert S., 2013, « La résilience des formes. La ceinture urbaine de Paris sur la rive droite ", Études rurales, 191, p. 193-220.

SCHWien J.-J., 1992, Strasbourg. Document d'évaluation du patrimoine archéologique urbain, Paris, Tours, Ministère de la Culture, Centre National d'Archéologie Urbaine, 285 p.

SеYвотн A., 189o, Das alte Strassburg vom 13. Jahrhundert bis zum Jahre 1870. Geschichtliche Topographie nach den Urkunden und Chroniken bearbeitet. Text., Strasbourg, 329 p.

XANDRY C., 2013, Organisation d'un territoire aux abords de la ville. Le cas de Metz, Strasbourg et Reims du milieu du Moyen Âge au début de l'époque moderne, thèse de doctorat soutenue à l'Université de Strasbourg, $571 \mathrm{p}$. 